\title{
TORI Axioms and the Application in Physics
}

\author{
Dmitrii Kouznetsov \\ Institute for Laser Science, University of Electro-Communications, Chofu, Japan \\ Email: dima@ils.uec.ac.jp,DmitriiKouznetsov@gmail.com
}

Received June 12, 2013; revised July 15, 2013; accepted August 9, 2013

Copyright @ 2013 Dmitrii Kouznetsov. This is an open access article distributed under the Creative Commons Attribution License, which permits unrestricted use, distribution, and reproduction in any medium, provided the original work is properly cited.

\begin{abstract}
TORI refers to the Tools for Outstanding Research and Investigation; the site with this name has been available since March 2011 to February 2013 at http://tori.ils.uec.ac.jp; the clone is available at http://mizugadro.mydns.jp/t. TORI is based on 6 axioms; any scientific concept is postulated to have the following properties: applicability, verifiability, refutability, self-consistency, principle of correspondence and pluralism. The examples of application in physics are suggested.
\end{abstract}

Keywords: Laws of Conservation; Fundamental Principles

\section{Introduction}

This article describes methodology used to built-up the special scientific site called TORI [1], which is abbreviation of Tools for Outstanding Research and Investigation. Since 2011 March to 2013 February 27, the site was available at http://tori.ils.uec.ac.jp/TORI [2]. The aggressive removal of that site from the free access indicates the importance of the problems touched there. Then, the clone of TORI is arranged at http://mizugadro.mydns.jp/t [3]. The main principles of TORI are presented in this article.

The article is organized in the following way. Section 2 lists the axioms that are postulated as criteria to distinguish Science from other knowledge (religion, art, customs). Section 3 indicates, that the TORI axioms are not so new as they may look. Section 4 declares the satisfaction of the personal curiosity of researchers as main goal of Science. The TORI axioms appear as justification of this goal. Section 5 provides basic examples of application of the TORI axioms in Optics. Section 6 suggests more examples of application of the TORI axioms. Section 7 discusses the future improvement of the methodology. Section 8 suggests the future ways of practical use of the TORI axioms.

\section{Axioms}

TORI appears as scientific philosophy (and, perhaps, even scientific religion), based on 6 dogmas, postulates, listed below. These postulates, or axioms, make difference between Science and pseudoscience, between science and various religions. TORI deals with concepts, satisfying the following axioms:
1) Applicability: The concept has some limited range of validity, distinguishable from the empty set.

2) Verifiability: In the terms of the already accepted concepts, some specific experiment with some specific result, that confirms the concept, can be described.

3) Refutability: In the terms of the concept, some specific experiment with some specific result, that negates the concept, can be described.

4) Self-consistency: No internal contradictions of the concept are known.

5) Principle of correspondence: If the range of validity of a new concept intersects the range of validity of another already accepted concept, then, the new concept either reproduces the results of the old concept, or indicates the way to refute it. (For example, the estimate of the range of validity of the old concept may be wrong).

6) Pluralism: Mutually-contradictive concepts may coexist; if two concepts, satisfying axioms $1-5$, have some common range of validity, then, in this range, the simplest of them has priority.

These axioms were suggested in the Russian journal "Uspekhi" [4] (in Russian) and in the First issue of the "Far East Journal of Mechanical Engineering and Physics" [5]. Then, the special site, TORI, had been designed to collect and develop concepts, based on axioms 1-6 above. Since that, these axioms can be referred as "TORI axioms".

\section{Axioms TORI Are Not New}

Axioms 1 - 6 above are not so original. Similar ideas had been declared by various researchers in century 20 and 
even before. Since the postulating of the 6 axioms above at $[1,3,4,5]$ and presenting them at the Sugadaira summer school [6], I got many questions and suggestions to reduce, simplify the set of axioms. The need of each of them is indicated below.

Statement of Axiom 1, that the range of validity of the concept should be limited, had been suggested to withdraw from the First axiom. Practically, this refers to extension of the range of validity of some concept to the Full Set. Empirically it is known, that the consideration of elements of the Full Set leads to contradictions; for example, the "Barber paradox": The barber in the military corpus is ordered to shave everybody, who does not shave himself: Should he shave himself?. Generalizing this paradox, the "barber" should be replaced to "set", and "shave" should be replaced to "include as element". In order to avoid such paradoxes, the limit of applicability is important. Especially dangerous is consideration of any concept that includes itself into area of applicability. For this reasons, while possible, I do not treat the TORI axioms, by themselves, as a scientific concept. Let this set of axioms be considered as some special kind of religion; then, the only applications of these axioms in the research activity should be qualified as "Science" (but never the elaboration of these axioms). Then, the barber, mentioned above, will never have need even to think about shaving himself.

Need of verifiability (Axiom 2) comes from many philosophies; in particular, the idea to recognize the tree by its fruits is specified in the Bible; if some tree is declared to be fruitful, let it bring at least one fruit. Verifiability seems to be the oldest principle of science.

Requirement of refutability (Axiom 3) had been suggested by Karl Popper [7-9]. Concept, that does not allow its refutation, cannot predict any non-trivial phenomenon: any result of any experiment, observation, calculus can be interpreted in favor of the concept. Popper uses term "falsifiability", that may have different (and sometimes opposite) meaning; in particular, at the transliteration to other languages. For this reason, term "Refutability" is used instead.

Importance of Axiom 4 (self-consistency) can be seen from the analysis of the official methodology that was compulsory in the USSR. The dialectics had been postulated as the base of the "omnipotent" and the only true Marx theory. Then, with this pretext, many branches of Science were attacked (Theory of relativity, Quantum mechanics, Genetics, Cybernetics, etc.). With Axiom 4, no dialectics, no doublethink [10] is allowed in any scientific concept; two independent researchers applying the concept to some case are supposed to come the same predictions. In order to avoid phenomena, typical for the USSR and described by Orwell, the self-consistency is declared in the special axiom.
The correspondence principle (Axiom 5) had been suggested by Niels Bohr [11], aiming the application to Quantum Mechanics; however, the principle is much more general and should be applied to other concepts too.

Axiom 6 (pluralism) is necessary to protect the new, alternative concepts, while they are not yet developed sufficiently to get status of principal; the tolerance with respect to the new concepts allows the scientific revolutions considered by Thomas Kuhn [12].

\section{Goal of Science}

In TORI, the human knowledge, based on axioms above, is qualified as Science. The main goal of science is declared to be satisfaction of the personal curiosity of the researchers. Then, the TORI axioms provide the apology, the excuse, the justification, why the money of the taxpayers should be spend for the researcher's curiosity. The researches that do not satisfy the TORI axioms, contrary, should not get foundation from the taxpayers.

Observations of development of pseudoscientific projects indicate, that the curiosity is the key point of any scientific research; there is no other way to make science. Some projects, initially created as scientific, have to continue, in order to provide the foundation for the colleagues employed, even if the need to reconsider the main doctrine of the project becomes evident. For example, the big projects of building of the nuclear fusion power plant on the base of laser ignition of targets continued long after the failure of these projects become evident. The continuation of those projects had been justified by the needs of the industry. Generally, the attempts to submit Science to other, more practical purposes, causes its profanation; it converts to the business of milking the budget, and leads to the frauds; and the colleague, being told about the new scientific problem, ask first the economical question: "What is my personal profit in this activity?" In some cases, the main goal of science above overlaps with economical interests of the business persons and administrators; in these cases, the certain branch of science, certain scientific projects get huge financial support. Often, this support replaces the curiosity, becomes the main goal, killing the initially scientific orientation of the project.

The TORI axioms help to reveal the scientific frauds, but also to catch the unwanted errors. In such a way, the TORI axioms push the research into development of new, "outstanding" concepts, that can be verified, revised, used, and easy refuted as soon as a new, more efficient concept happens to be valid in the same range of applicability.

The TORI axioms appear as empirical guess; it is wain to try to "prove" them. They cannot be proven; the only their efficiency in the research can be compared to that of another systems of postulates. The efficiency of TORI 
axioms can be compared to that of the Marx-Lenin philosophy (that was compulsory in the USSR and had suggested many predictions that happened to be just wrong). The efficiency of TORI can be compared to that of dogmas of Christians (which did not prevent them from crusades, nor from the inquisition, nor from participation of christians at both sides of the front line during the World Wars). Results of application of TORI can be compared to any system of dogmas of any other religion, methodology, philosophy.

According to declaration above (not to apply TORI to themselves), the comparison of efficiency of TORI to that of other systems of concepts falls out of competence of TORI; therefore, this comparison is not considered here anymore.

\section{Laser Science}

Misinterpretations, discussions, conflicting concepts exist in any science, and the Laser Science is not exception. In this section, few examples are mentioned, in which the methodology TORI (even while it had not yet been declared) had been used to defend the correct, self-consistent interpretation and/or to refute some results as "wrong”. In order to avoid citing of a hundred of references, below, the only my articles with the solutions are mentioned; the criticized articles are cited there.

The idea of quantum consideration of the optical solitons had been considered in century 20. The photon wave function, that can be interpreted as a classical soliton, had been constructed. Two colleagues had calculated the time evolution of the distribution of the intensity operator; they found that this distribution spreads with time. On the base of this observation, they made conclusion, that the quantum fluctuations annihilate the optical soliton. It took serious efforts to show that such a concept is wrong.

The main difficulty was, that initially, the statement about quantum annihilation had not been formulated as a scientific concept, namely, neither ways of verification nor ways of the refutation had been indicated. Roughly, the annihilation means that parts of the soliton (id est, photons or clusters of photons) can be found, registered at large mutual separation, say, big in comparison to the width of the initial soliton. Inability to detect such separated particles can be interpreted as sufficient evidence for refutation of the concept of the quantum annihilation. The detection of separated (un-coupled) photons corresponds to the four-order correlation for the photon wave function. Then such a correllator had been calculated [13, 14] (and happened to be stable); the concept of quantum annihilation of the optical soliton had been refuted. Now the concept of quantum stability of the idealized optical soliton is accepted by the scientific community.

In century 20, talking about Quantum Optics, it was convenient to consider the quantum oscillator. Such phenomena as squeezing and the fractal revival of the quantum wave packets had been discussed. The concept of the optical filed as quantum oscillator was supposed to be valid in all cases. The limits of description of the light with a single mode (that is in certain sense equivalent to a harmonic oscillator) were not even mentioned. The use of the First axiom of TORI would reveal this fault. The estimates of the efficiency of the excitation of other modes by the simple nonlinearity [15] had revealed that the single-mode approximation is quite limited, at least, for the traveling waves and pulses. Some squeezing can be achieved in such a configuration, but no fractal revival is possible: the state of photons in the chosen mode becomes entangled with states of field in other modes. This entanglement dominates; at the consideration of a single mode, it appears as thermal noise that is much stronger than the effect expected. In such a way, no fractal revival can be achieved in traveling wave. Indeed, during 15 years since that prediction, no fractal revival of the quasi-classical wave packets in the traveling wave had been reported. The range of applicability of the description of light as quantum oscillator happens to be very narrow.

The idea of combining of light guided in optical fibers, at the appropriate splicing and splitting, revives again and again in various countries. The increase of the brightness had been expected for the multimode optical pump at the linear combining of fiber delivering the pump (Arizona, 2003). The real-time discussion had prevented the publishing of such a concept. Indeed, the concept does not satisfy the Axiom 5 (principle of correspondence): the range, where one still can use the Liouville Theorem, that prohibits the increases of brightness in linear optical systems, had not been indicated. The careful measurement confirmed, that the combining of guided light enhances the numerical aperture, occupied by the beam; this sets the natural limit of the combing.

Another application of the correspondence principle refers to the wonderful properties of the Yb-doped Gadolinium-based ceramics. Many curves of the efficient cross-sections of the the laser transition had indicated the strong violation of the McCumber relation. No indication to narrowing of limits of applicability of the McCumber relation had been supplied in the articles that promoted the new laser material. On the base of the correspondence principle (axiom 5), the curves published in various journals were qualified as errors [16]. In that specific case, the mistake had been attributed to the re-absorption; the samples were not optically thin, as the treatment of the primary data implied.

Recently, the formalism of superfunctions [17-25] had beed suggested, that allows to deal with the opticallythick samples and still recover the local properties of the 
laser materials [24]; the improvement of precision of characterization of optical materials is expected with this formalism.

The lack of pluralism (Axiom 6) made difficulties in discussion and promotion of the interpretation of the quantum reflection from the ridged mirrors in terms of the Zeno effect. The only simplicity of the formula suggested and the tremendous agreement with the fresh data for quantum reflection of He atoms saved the new concept, and allowed the publication of the corresponding theory $[27,28]$.

Perhaps, any researcher had observed the wrong publications, statements, presentations, where the mistake could be revealed with axioms TORI, without to drill through the deduction, nor dig deep into the experimental set-up. Usually, there is no need to declare the publication as "wrong", it is sufficient to indicate, which basic principle should be revised on the base of the new result. I suggest only one example: "Medium with such a wonderful properties is very interesting. It should allow to make not only new efficient laser, but also the perpetual motion machine of Second kind." Usually, the authors are not ready to negate the fundamental principles, and consider such a diplomatic construction as kind of accusation, and sometimes found mistakes in their deductions or experiments.

After to get some new result, the researcher is supposed to formulate it as refutable concept, and try to refute it by himself. At the Bisson-effect (Switching of emissivity and photoconductivity in Yb-doped ceramics), the pump light is converted to the emission of white light, if the pump power exceeds some threshold value [29]. The initial estimate of size of the pumped spot and the total amount of power converted to the white visible light leaded to estimate of the effective temperature. This effective temperature happened to be well above the temperature of melting of the sample. That estimate leaded to the conjecture, that the new quantum phenomenon takes place, the multi-photon excitation of electrons into the conduction band, weakly coupled to the vibrational degrees of freedom.

The attempts to verify such a conjecture were done: Electrons in the conduction band are supposed to convert the dielectric to, as minimum, some kind of semiconductor. Indeed, the raise of conductivity for many orders of magnitude had been detected at the avalanche of the broadband light emission. In this sense, the verification (TORI axiom 2) had been successful.

Also, we tried to refute (Axiom 3) the concept. If the electrons in the conduction band are uncoupled from the vibrational degrees of freedom, then the effect should be stronger at low temperatures, while no phonons affect the up-conversion of light by electrons. Merging of the sample into a liquid happened to be sufficient to eliminate the effect. In such a way, the concept about the new beautiful quantum effect had been refuted. The broadband emission can be attributed to the heating of the sample, that enhances the absorption of the pump, converting it to the thermal radiation. In such a way, the promising concept had been refuted at very beginning. Even if the non-termal component of the Bisson-effect exists, it is difficult to extract it at the background of the banal heating of the sample and the corresponding thermal emission. In the similar way, the jump of the electric conductivity of dielectrics can be attributed to the raise of the temperature and corresponding re-structuring of the surface of the sample.

The cases above show the examples of application of the TORI axioms to the Laser Science. However, the general principles of TORI (Except, the idea of refutability by Karl Popper) were not declared that time. Contrary, the TORI axioms were formulated as generalization of that experience. In this sense, the TORI axioms appear as empiric observation, as simplification of the practical activity of treating and analyzing scientific (and not so scientific) results.

\section{Not Only Laser Science}

Initially, the TORI axioms were supposed to describe concepts that deal with the Laser Science. However, their application is not limited to the Laser science. The two examples of application of the TORI axioms in other areas refer to the Mathematics of Computation and Complex Analysis, namely, evaluation of tetration [17-21] and other superfunctions [22-26] and to observation of processes that happen with science in Russia and, in particular, at Skolkovo [31,32].

Application of the TORI axioms to computational mathematics happened to be successful in many senses. The general formalism of superfunctions is constructed; the effective algorithms of the evaluation are implemented; many non-trivial superfunctions [25] are described [17, $19,20,22,23,26]$; the beautiful figures are plotted; even the use in the Laser Science is suggested [24,26].

The second attempt, about observation of decay of science in Russia, happens to be less successful. Many links and evidences in support of article [32] were collected at TORI; but 2013.02.27, within a week since the reviewers of "Physics Today" got the manuscript [32] for the evaluation, the site http://www.ils.uec.ac.jp/TORI had been attacked, the free reading access had been disabled [2], and it takes serious efforts to recover it.

Some colleagues suggest to set some kind of taboo on the scientific research of certain topics. However, this should be explicitly declared, for example: "Our goal is future degradation of culture, technology and science in Russia. Therefore, the scientific analysis of things that happen in Russia, and, in particular, with science in Rus- 
sia, should not be published". While such a declaration is not written, not accepted as Law, not published, Science in Russia should be considered as appropriate topic for the scientific research. Then, the attack 2013.02.27 on site TORI should be qualified just as barbarian vandalism.

An alternative solution could be just following the verbal orders of pseudo-scientists and avoiding discussion of any fraud in science, as A. Kaminskii suggests [30]. As a development of this idea, the prohibition to submit "Errata" and negative review reports could be considered. From my point of view, such an attitude would kill any science in a country within few generations of researchers.

\section{Discussion}

Often, the researchers believe that they present some kind of the "objective knowledge", or even some kind of the "absolute true". This can be expressed with sentences "Our results are true, and there cannot exist any experiment, contradicting our results" or "Why should I help competitors to refute our result?". The attempt to describe a gedanken experiment, that could refute the concept, or any alternative concept, is often interpreted as a personal offense, that justifies any reciprocal response (canceling of the opponent's presentation, the attack on the website of the opponent, etc.). Such an attitude should be qualified as pseudo-scientific and condemned. The axiom 3 (refutability) could mitigate this antagonism, converting science to the academic activity and promoting civilized ways of scientific discussions.

Purification of science from pseudoscience is not straightforward, because the scientific revolutions [12] should be allowed. The TORI axiom 6 defends the pluralism, allowing consideration of new, "crasy idea", an alternative concepts (under condition, that it satisfies axioms 1 - 5). The new idea may look pretty creasy. The Galvani effect (with frog's legs) looked nonsense. Violation of the fundamental concept of conservation of number of atoms of each kind in any closed system (at the discovery of radioactivity) did not look better. The negation of the universal time (in the special theory of relativity) and the absence of classical trajectories (in Quantum Mechanics) also give good examples of breaking rules, that seemed to be "absolute true". Even concept about failure of axioms of arithmetics (existence of some "Mizugadro number" as biggest integer, for which the axioms of arithmetic begin to fail), in principle, could be treated as scientific, as soon as one suggests any way to refute such a concept. The TORI axioms are ready for very strong scientific revolutions, still allowing to identify and reveal the pseudo-science, frauds and mistakes.

The need to fight pseudoscience is not obvious. Alek- sander Kaminskii thinks, that it is vain to fight against the pseudo-science, and it is better to allow the pseudo-scientists to get grants and to spend them to some fake researches, than to reveal the wrong publications [30]. However, the scientific consideration should at least honestly declare, what is happening to the Science. The TORI axioms give the formal criteria to distinguish science from magia, ufology, astrology, scientology, politics, religions, and other kinds of knowledge, that may look similar to science.

For the beginning of century 21, the TORI axioms seem to be most formal criteria to separate Science among other kinds of the Human knowledge and Human activity. However, as soon, as some new, more efficient criteria will be suggested, the TORI axioms will have to be replaced with a more modern and efficient philosophy.

\section{Conclusions}

The 6 axioms TORI are formulated. These axioms appear not only as a tool for the efficient research, but also as justification of the main goal of science as satisfaction of curiosity of researchers. I think the TORI axioms should be declared as primary and main requirements for all manuscripts submitted for the publication in a scien- tific journal.

Axiom 3 (Refutability) seems to be the most important for the beginning of 21st century. Each article should indicate, in which case (new deduction, simulation, observation, experiment, measurement) the authors should confess: "Sorry, our concept is wrong". Only if some ways of the refutation exist, the non-trivial predictions can be done on the base of the results presented.

\section{Acknowledgements}

Most of content of this article had been available at [1]. This article is inspired by Shigeaki Nishio, Hitoki Yoneda, Akira Shirakawa, Mitsuru Musha, Takashi Mukaiyama, Hajime Nishioka, Ken-ichi Nakagawa, Nobuyuki Nakamura, and Makoto Morinaga, who had disabled the access to tori.ils.uec.ac.jp/TORI, where the TORI axioms are described.

\section{REFERENCES}

[1] D. Kouznetsov, "Tools for Outstanding Research and Investigation,” March 2011-February 2013. http://tori.ils.uec.ac.jp/TORI

[2] D. Kouznetsov, “Tori Attacked,” 2013. http://samlib.ru/k/kuznecow_d_j/toriattacked.shtml

[3] D. Kouznetcov, "Mizigadro, Tools for Outstanding Research and Investigation,” 2013. http://mizugadro.mydns.jp/t

[4] D. Kouznetsov, "Place of Science and Physics in Human 
Knowledge,” Uspekhi, Tribune, 2010-2011, No. 110. Official Russian version: http://ufn.ru/tribune/trib120111. English versions:

http://samlib.ru/k/kuznecow_d_j/2010mestoe.shtml http://mizugadro.mydns.jp/t/index.php/Place_of_science_ in_the_human_knowledge

[5] D. Kouznetsov, Far East Journal of Mechanical Engineering and Physics, Vol. 1, 2010, p. 1. http://mizugadro.mydns.jp/PAPERS/2010support.pdf

[6] D. Kouznetsov, "How to Write Scientific Papers in English,” Sugadaira Summer School, 2012. http://www.ils.uec.ac.jp/ dima/CONF/2012sugadaira.pdf

[7] K. Popper, "Science: Conjectures and Refutations, Philosophy of Science: A Personal Report," In: C. A. Mace, Ed., British Philosophy in Mid-Century, 1957.

http://philosophyfaculty.ucsd.edu/faculty/rarneson/Course s/popperphil1.pdf

[8] K. R. Popper, "The Logic of Scientific Discovery,” Basic Books, Oxford, 1959.

http://psycnet.apa.org/psycinfo/1961-02882-000

[9] K. R. Popper, "Science as Falsification. Karl Popper, Conjectures and Refutations," Routledge and Keagan Paul, London, 1963, pp. 33-39.

http://www.stephenjaygould.org/ctrl/popper_falsification. html

[10] G. Orwell, “1984,” 1949. http://www.george-orwell.org/1984

[11] A. Bokulich, “Bohr’s Correspondence Principle,” 2010. http://plato.stanford.edu/entries/bohr-correspondence/

[12] T. S. Kuhn, "The Structure of Scientific Revolutions," 2nd Edition, University of Chicago Press, Chicago, 1970. http://www.des.emory.edu/mfp/Kuhn.html

[13] D. Yu. Kouznetsov, Quantum Optics, Vol. 4, 1992, pp. 221-227. http://iopscience.iop.org/0954-8998/4/4/003/

[14] D. Yu. Kuznetsov, JETP Letters, Vol. 54, 1991, pp. 568570. http://www.jetpletters.ac.ru/ps/1266/article_19153.pdf

[15] D. Kouznetsov and E. Gomez, Optics and Spectroscopy, Vol. 87, 1999, pp. 594-602. http://mizugadro.mydns.jp/PAPERS/gomez.pdf

[16] D. Kouznetsov, Chinese Optics Letters, Vol. 5, 2007, pp. S240-S242. http://col.org.cn/abstract.aspx?id=COL05S1S240-3

[17] D. Kouznetsov, Mathematics of Computation, Vol. 78, 2009, pp. 1647-1670.
doi:10.1090/S0025-5718-09-02188-7

[18] D. Kouznetsov, Vladikavkaz Mathematical Journal, Vol. 12, 2010, pp. 31-45.

http://www.emis.ams.org/journals/VMJ/articles/2010_2_ 4.pdf

[19] D. Kouznetsov and H. Trappmann, Mathematics of Computation, Vol. 79, 2010, pp. 1727-1756. doi:10.1090/S0025-5718-10-02342-2

[20] H. Trappmann and D. Kouznetsov, Mathematics of Computation, Vol. 81, 2012, pp. 2207-2227. doi:10.1090/S0025-5718-2012-02590-7

[21] H. Trappmann and D. Kouznetsov, Aequationes Mathematicae, Vol. 81, 2011, pp. 65-76. doi:10.1007/s00010-010-0021-6

[22] D. Kouznetsov, Moscow University Physics Bulletin, No. 65, 2010, pp. 91-98. doi:10.3103/S0027134910020049

[23] D. Kouznetsov and H. Trappmann, Moscow University Physics Bulletin, Vol. 65, 2010, pp. 6-12. http://www.springerlink.com/content/qt31671237421111

[24] D. Kouznetsov, Optical Review, Vol. 20, No. 4, 2013, pp. 321-326. doi:10.1007/s10043-013-0058-6

[25] http://en.citizendium.org/wiki/Superfunction

[26] D. Kouznetsov, “Tania Function and Doya Function,” 2013. http://mizugadro.mydns.jp/t/index.php/Tania_function

[27] D. Kouznetsov and H. Oberst, Physical Review A, Vol. 72, 2005, Article ID: 013617. doi:10.1103/PhysRevA.72.013617

[28] D. Kouznetsov and H. Obrest, Optical Review, Vol. 12, 2005, pp. 363-366. doi:10.1007/s10043-005-0363-9

[29] J.-F. Bisson, D. Kouznetsov, K. Ueda, S. T. FredrichThornton, K. Petermann and G. Huber, Applied Physics Letters, Vol. 90, 2007, Article ID: 201901. doi:10.1063/1.2739318

[30] A. Kaminskii, "It Is Vain to Fight the Wrong Publications. It Is Getting Worse and Worse, and Nothing Can Be Done about This. We should Make Science while Some Fonds Are Still Assigned to Science, while the Pseudo-Scientists Have Not yet Have Occupied Everything," Private Communication, 2008.

[31] T. Feder, Physics Today, 2013. doi:10.1063/PT.3.18500

[32] D. Kouznetsov, “Corruption in Russian Science,” 2013. http://mizugadro.mydns.jp/PAPERS/2013physToday.pdf 